\title{
A LUNAR RADAR STUDY AT Io-CM WAVELENGTH
}

\author{
B. S. YAPLEE, NANCY G. ROMAN, K. J. CRAIG, AND T. F. SCANLAN \\ Radio Astronomy Branch, U.S. Naval Research Laboratory \\ Washington, D.C., U.S.A.
}

In February 1957 the U.S. Naval Research Laboratory was successful in obtaining radar echoes from the moon by using a short-pulse radar [1]. Although the signal-to-noise ratio was not as large as desired, the leading edge of the echo was sufficiently well defined to make possible an accurate measurement of the radar distance to the moon. It was hoped that geodetic information could be extracted from such distance measurements. Hence in October and November, 1957, the radar distance to the moon was measured with a radar mounted in the 50 -foot steerable parabola at NRL.

Two-microsecond pulses with a peak power of 2 megawatts and a carrier frequency of $2860 \mathrm{Mc} / \mathrm{s}$ were transmitted at intervals of 4 milliseconds. The ranging accuracy of this system was of the order of one-fifth of a mile. The radar echoes were displayed on an A-scope and recorded photographically on 35-mm film as shown in Fig. 1. Approximately 26 superimposed traces were photographed together with the time, the counter readings, and a data card. Data were recorded once each second for the first minute of each ten-minute interval. Since the first portion of the echo consisted of a few spikes that fluctuated slowly in and out of the noise background, the leading edge of the radar echo could not always be associated confidently with the center of the lunar disk. To obtain greater accuracy, the apparent variation in range of the leading edge of the radar echo was read and plotted against the time of observation. A sample plot is shown in Fig. 2. An echo delay time was read from near the middle of each plot, after the slopes of successive plots were checked to see that smooth velocity and acceleration curves resulted. Comparisons of the radar distances obtained in this manner with the calculated distances are shown in Figs. 3 to 9. The calculations were based on the geometry of the earth-moon system as shown in Fig. 10. Fig. 11 illustrates the average residual and the peak-to-peak variation of the residuals for each period of observation.

A significant feature of the residuals is that they are all systematically positive. This suggests that the value for either the earth's equatorial radius, the moon's horizontal parallax, or the moon's radius, is in error. In the equation of Fig. 10 the predominant term is $a / \sin \pi$, the center-to-center distance between the earth and the moon. Any error in the quantity $a$ (earth's equatorial radius) or in $\pi$ (moon's horizontal parallax) is magnified 50 to 70 times in the total calculated distance. Thus, an accurate measurement of the moon's distance should yield a more accurate value of the earth's equatorial radius 


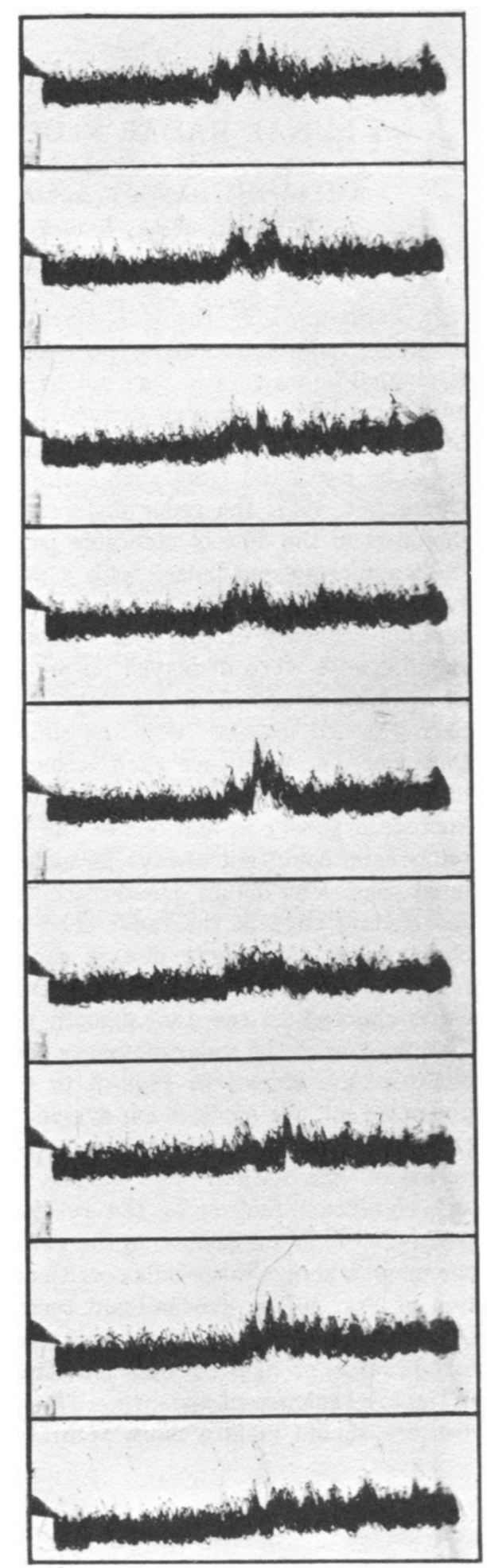

FIG. 1. Nine-second sample of recorded data with a 260-microsecond sweep. 


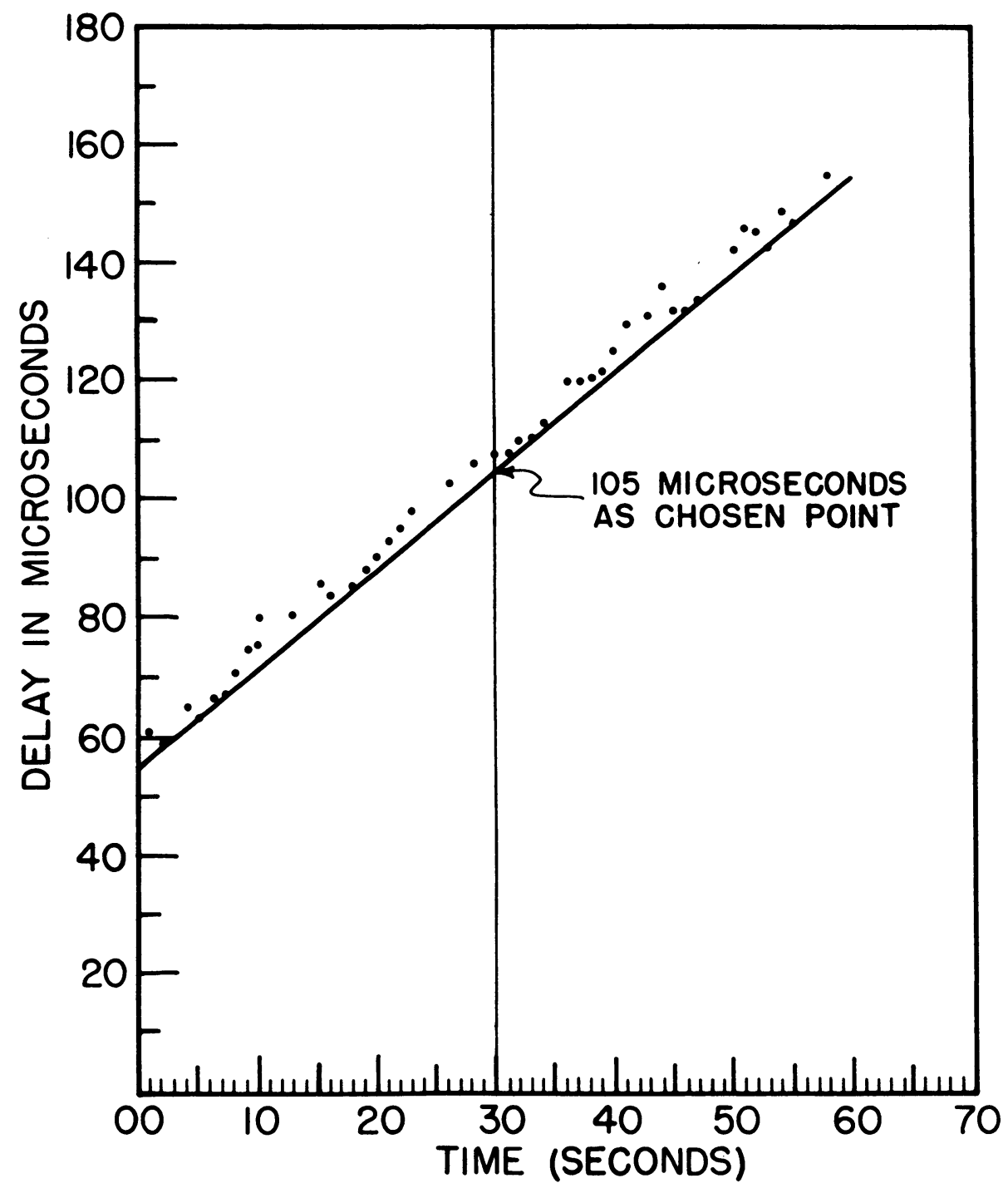

Fig. 2. Sample plot of the apparent leading edge of the moon echo for one minute of the recorded data.

if the values of $\pi$ are sufficiently accurate. This technique, however, has its difficulties. First, the backscattering areas on the moon deviate from an adopted mean surface. Second, the residuals may arise, at least partly, from inaccuracies in the value of the moon's horizontal parallax. Finally, uncertainties in other factors, such as the shape of the earth or the velocity of propagation in the medium between the earth and the moon, may account for part of the residuals. 

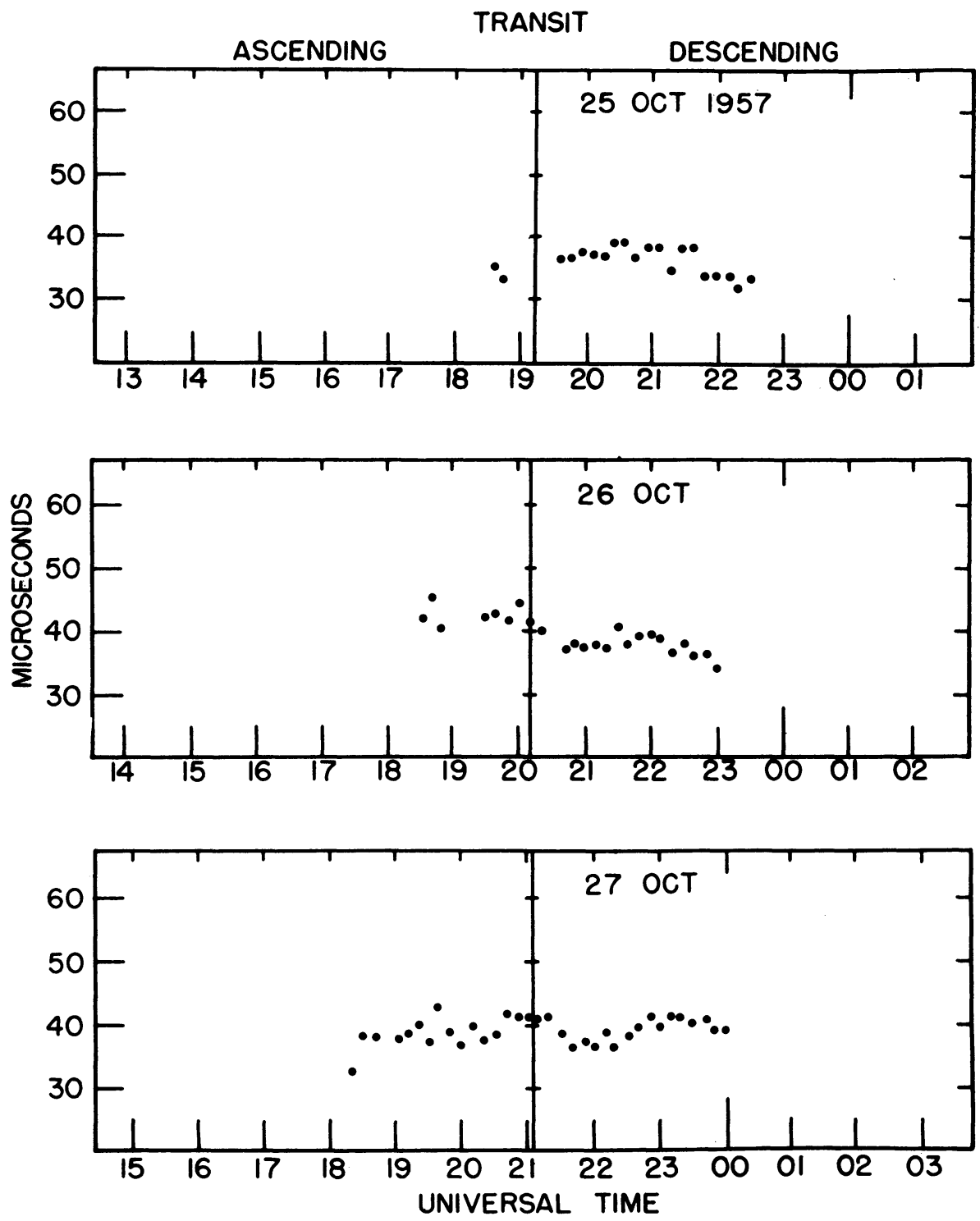

Fig. 3. Distance residuals (radar distance minus calculated distance). The residuals shown in Figs. 3 through 9 are subject to slight ephemeris-time corrections. 

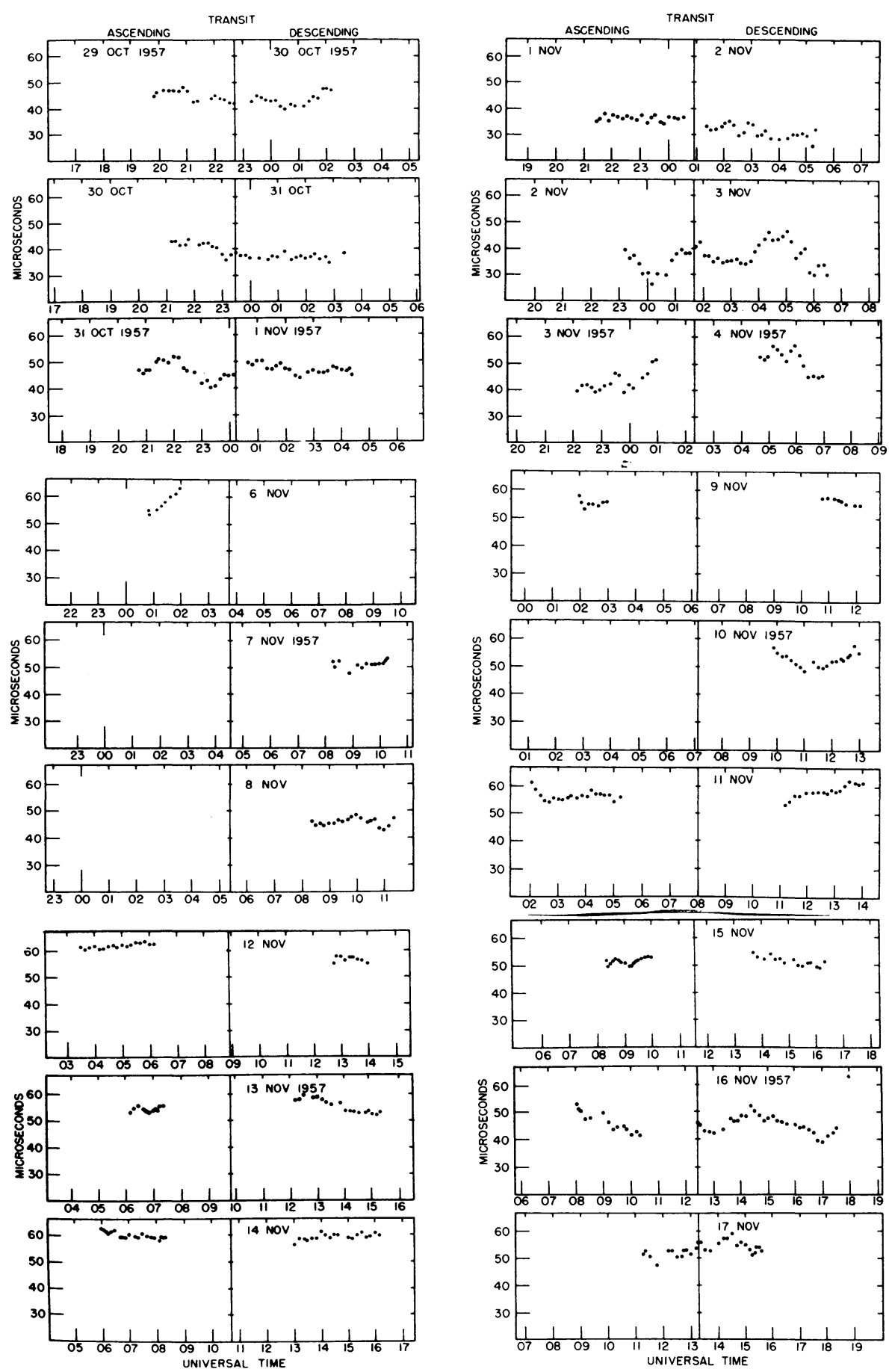

FIGS. 4-9. Distance residuals (radar distance minus calculated distance). 


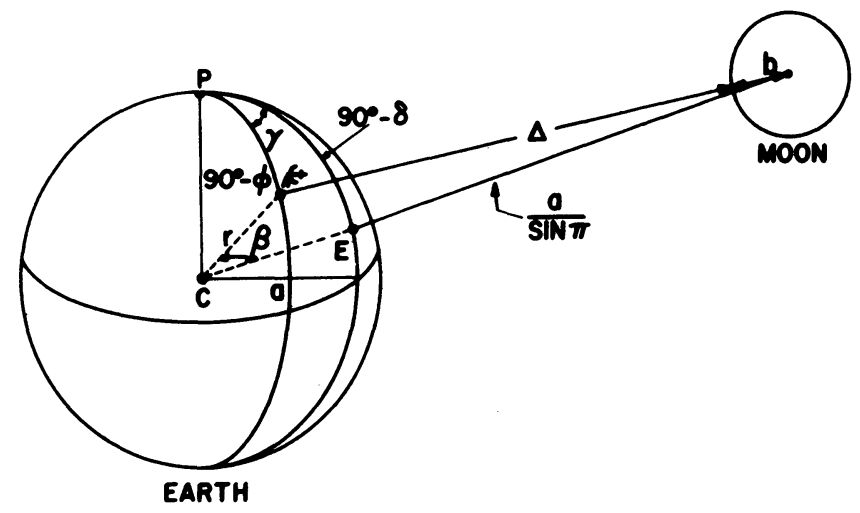

FIG. 10. Diagram of the earth-moon distance problem.

$$
\begin{aligned}
(\Delta+b)^{2} & =r^{2}+(a / \sin \pi)^{2}-(2 r a / \sin \pi) \cos \beta \\
\cos \beta & =\sin \phi \sin \delta+\cos \phi \cos \delta \cos \gamma \\
\Delta & =-b+\left[r^{2}+(a / \sin \pi)^{2}-(2 r a / \sin \pi) \cos \beta\right]^{t} \\
2 \Delta / c & =N X(\text { pulse repetition period) }+ \text { delay time } \\
c & =299,792.8 \mathrm{~km} / \mathrm{sec}, \text { velocity of light [2] } \\
a & =6,378,270 \mathrm{~m}, \text { equatorial radius of earth [3] } \\
b & =1,740,000 \mathrm{~m} \text {, equatorial radius of moon [4] } \\
r & =6,369,910 \mathrm{~m}, \text { radius of the earth at NRL [5] } \\
\phi & =38^{\circ} 37^{\prime} 599^{\prime \prime} 8 \mathrm{~N}, \text { geocentric latitude of NRL's } 50 \text {-foot antenna } \\
\lambda & =77^{\circ} 01^{\prime} 366^{\prime \prime} 7 \mathrm{~W} \text {, longitude of NRL's 50-foot antenna } \\
\pi & =\text { horizontal parallax of the moon [6] } \\
\gamma & =\text { hour angle of the moon } \\
\Delta & =\text { distance from observer to the surface of moon }
\end{aligned}
$$

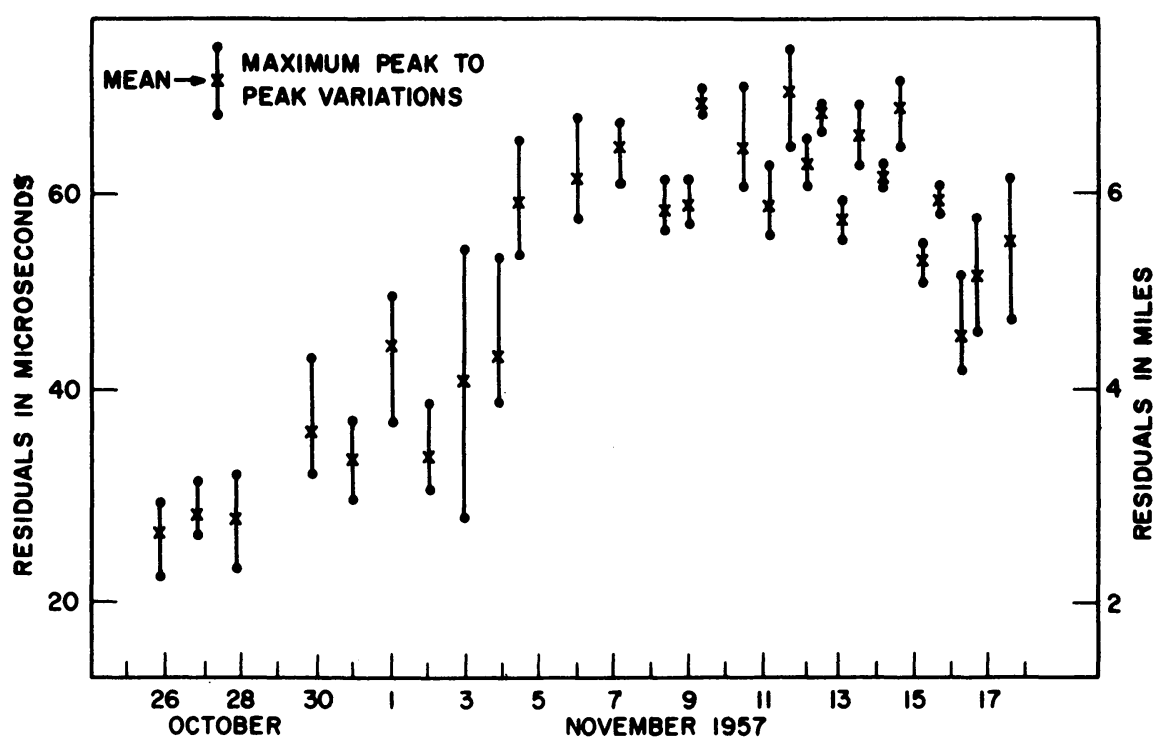

FIG. 11. Average residuals for each observation period. 


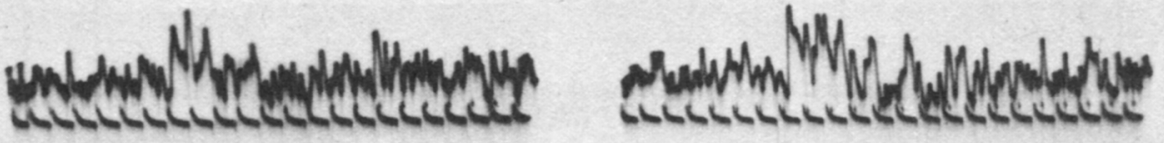

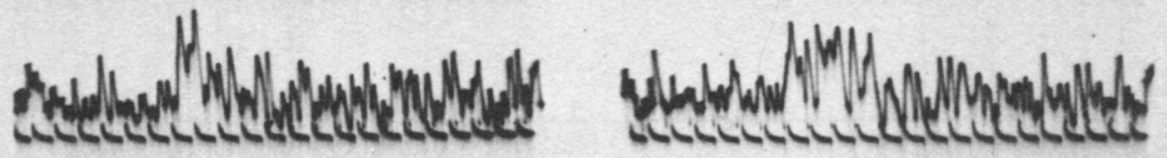

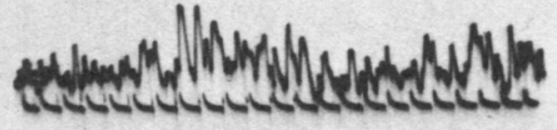

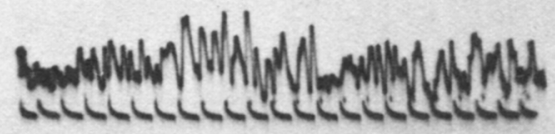

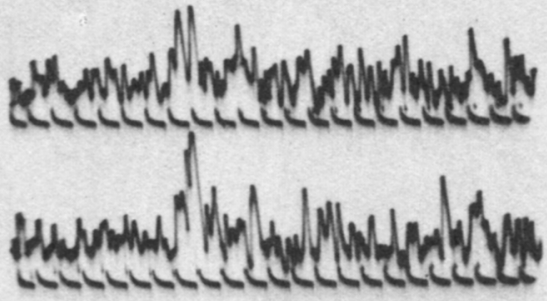

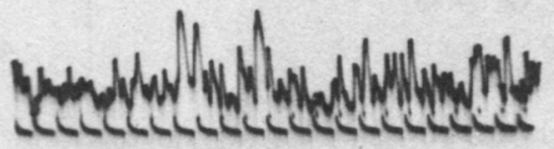

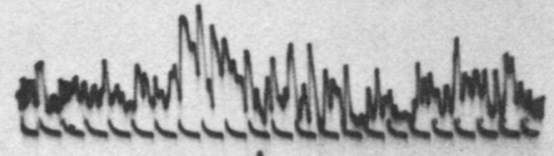

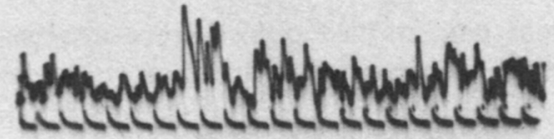

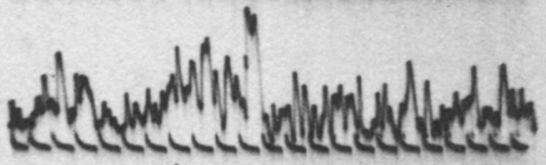

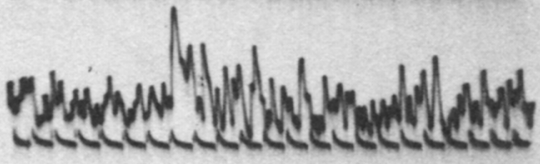

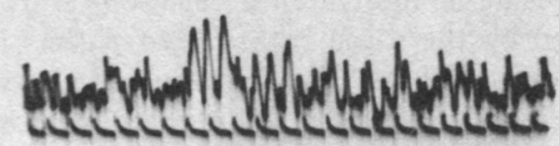

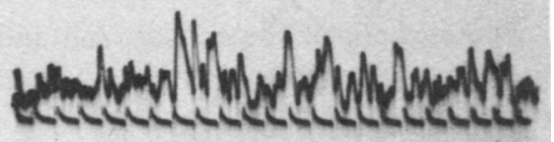

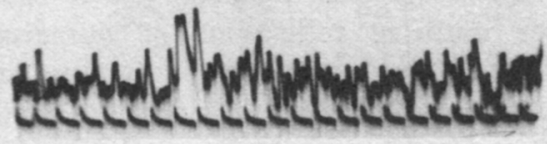

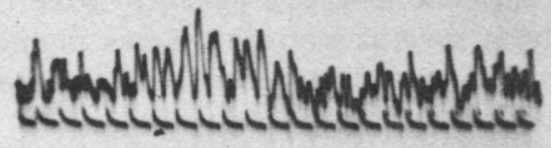

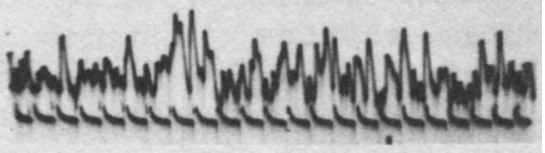

Fig. 12. Sample of fast film (range marks, 10 microseconds; trace separation, 4000 microseconds). 

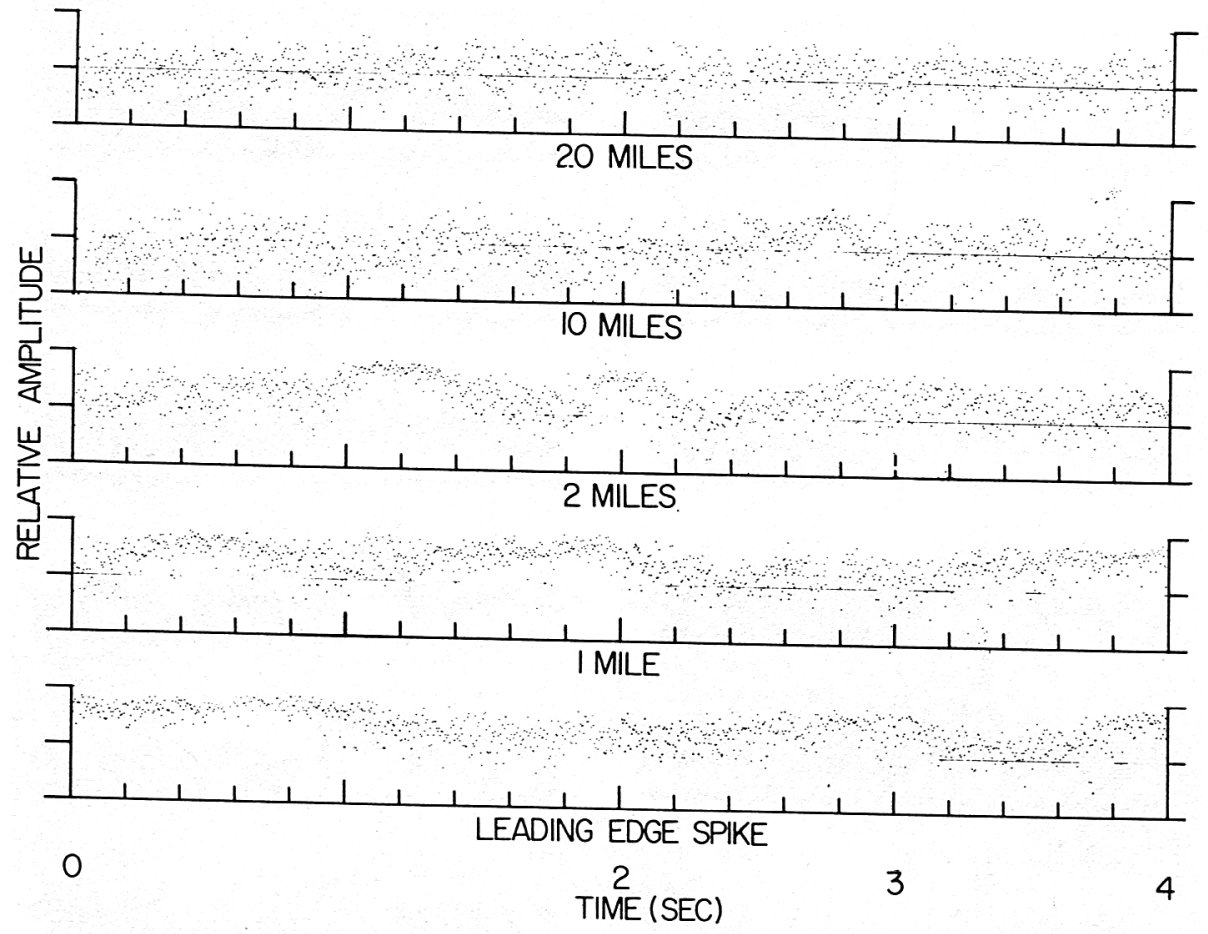

Fig. 13. Relative amplitude variations in time of radar echoes from moon.

Photographs of the echoes from individual transmitted pulses were analyzed to obtain information on the distribution of backscattering areas on the moon. A sample of the data is shown in Fig. 12. The fluctuating spikes of the composite echo are believed to be the result of interference between returns from several scattering areas illuminated by the same transmitted pulse. If each spike is a result of single reflections, a high degree of pulse-to-pulse correlation would be expected. Also, single reflections should produce a narrow frequency spectrum as the aspect of the moon with respect to the radar changes with time. Conversely, either multiple reflections or numerous discrete reflecting areas would produce a wider frequency spectrum and greater amplitude excursions. It is apparent from the records that the spikes show faster fluctuations as the samples increase in range from the leading edge of the echo. To strengthen this observation, the spike amplitudes have been analyzed at the leading edge and at range increments of 10, 20, 100, and 200 microseconds from the leading edge of the echo. Fig. 13 shows 1000 consecutive pulses (a four-minute sample) and their amplitude variations at the different ranges. An analysis of these pulses indicates that the amplitude distribution tends toward a Rayleigh distribution as the range at which the heights are sampled is increased (see Fig. 14). The autocorrelation functions of the pulse heights were also obtained (see Fig. 15). Fig. 16 shows the power spectra derived from the respective autocorrelation functions. From this pulseamplitude analysis, one can conclude that there is a high degree of pulse- 


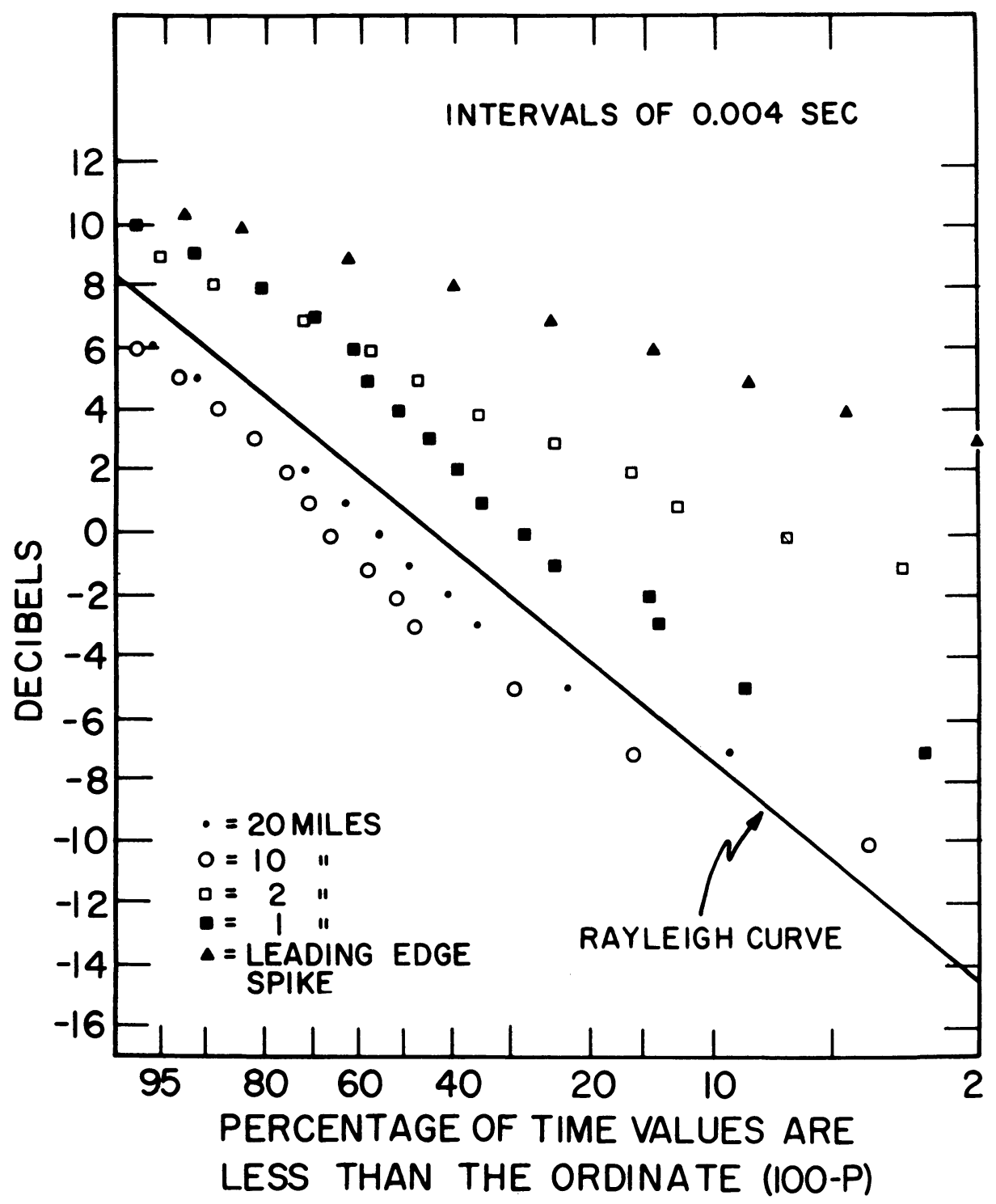

Fig. 14. Amplitude distribution at various ranges.

to-pulse correlation at the leading edge and at the immediately adjacent spikes, suggesting a single reflection from discrete areas. The spike amplitudes at greater ranges tend toward a random distribution and suggest multiple reflections or several reflecting areas at the same range. Further observations and analyses are necessary to confirm this result.

In summary, radar distance measurements to the moon can be made with a high degree of precision, but for geodetic purposes the difficulties outlined previously must be resolved. Work is in progress on the determination of 


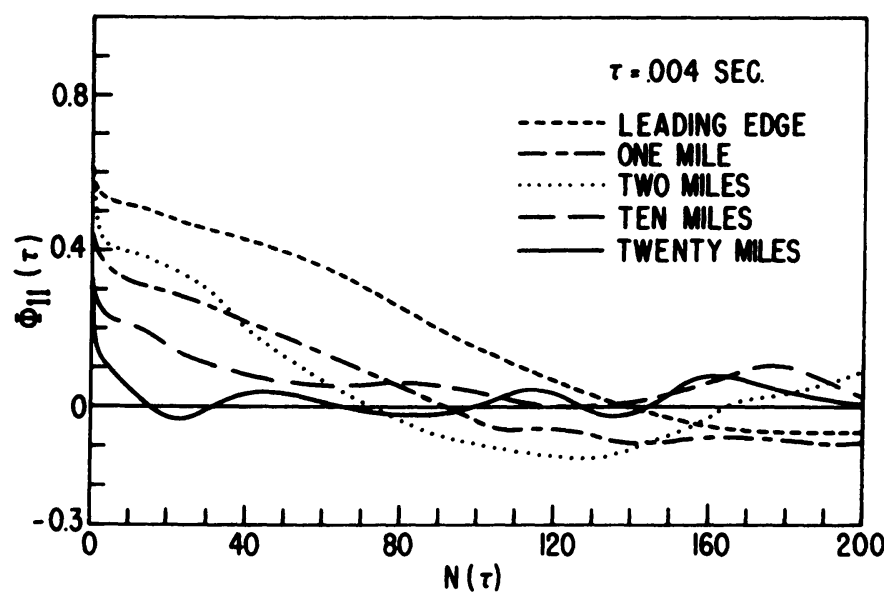

FIg. 15. Autocorrelation function for various ranges.

FIg. 16. Power spectra of radar echoes from the moon at various ranges.

a mean surface for the moon (the term $b$ in Fig. 2). Further studies will be made of the separation in the residuals of the effects of the earth's equatorial radius and the moon's horizontal parallax, as well as of the distance of the NRL radar from the axis of rotation of the earth.

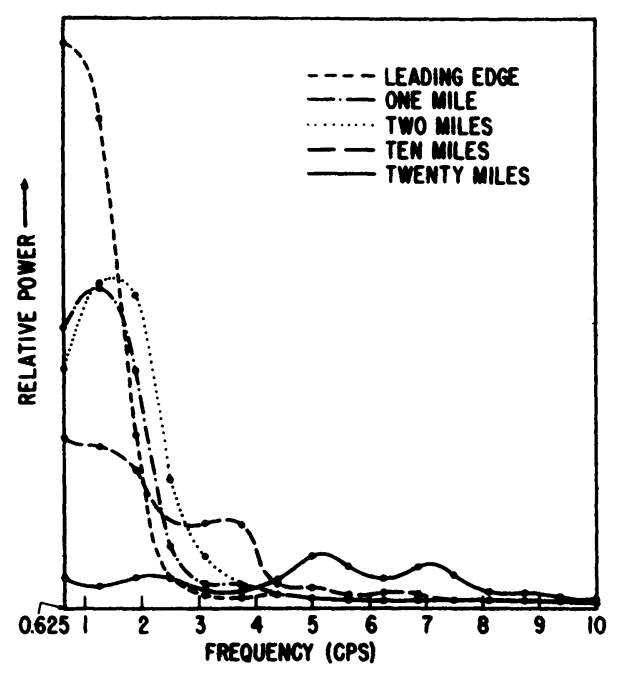

\section{REFERENCES}

[1] Yaplee, B.S., Bruton, R.H., Craig, K. J., and Roman, N. G. Proc. I.R.E. 46, 293, 1958.

[2] Mulligan, J. F., and MacDonald, D. F. Amer. J. Phys. 25, 180-92, March 1957.

[3] Obtained from the U.S. Army Map Service.

[4] Value obtained from C. W. Allen, Astrophysical Quantitios. London (The Athlone Press), 1955.

[5] Calculated from a formula in The American Ephemeris and Nautical Almanac, Washington, D.C. (Government Printing Office).

[6] Taken from Improved Lunar Ephemeris, 1952-1959. Washington, D. C. (Government Printing Office). 